TPeriodica Polytechnica Electrical Engineering and Computer Science

60(4), pp. 217-222, 2016

DOI: 10.3311/PPee.9686

Creative Commons Attribution (i)

\section{Innovative Methods in Activation Process of Through-hole Plating}

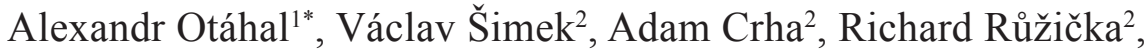 \\ Ivan Szendiuch $^{1}$
}

RESEARCH ARTICLE

\begin{abstract}
This paper is dealing with an in-depth verification of innovated activation methods, where the phase of surface treatment involves solvent with oxides of carbon as the core chemistry. The overall impact of solvent on through-hole copper plating process was tested on target $t$ structures involving standard FR-4 PCB substrate. The novelty of the approach proposed here is based on the effect of an unusual combination of vacuum and ultrasound waves in order to enhance quality of the activation process. The results undoubtedly demonstrated significant benefits of vacuum and vacuum/ultrasound combination on the decreasing number of defects, which normally occurred during the conventional plating process. In addition, there was also discovered that the resulting number of failures was increased even despite the use of ultrasound.
\end{abstract}

\section{Keywords}

plated-through holes, PCB activation, reverse pulse plating, vacuum, ultrasound

\footnotetext{
${ }^{1}$ Department of Microelectronics, Brno University of Technology,

Technická 3058/10, Královo Pole, 61600, Brno, Czech Republic

${ }^{2}$ Department of Computer Systems, Brno University of Technology, Božetěchova 2, Královo Pole, 61266, Brno, Czech Republic

*Corresponding author, e-mail: xotaha00@stud.feec.vutbr.cz
}

\section{Introduction}

Nowadays, many diverse methods have been used for the purposes of through-hole copper plating in the series production, as well as in the prototyping domain. Copper plating could be achieved by the method of direct current plating, pulse plating or reverse pulse plating. For a small-scale production and a rather small diameter of the drilled holes, there is commonly used pulse or reverse pulse plating $[1,2]$. Switchable reverse pulse plating produces more uniform copper deposition even for difficult aspect ratios and smaller holes. Reverse pulse plating is also very useful for small holes on high-density PCBs and improves the production reliability during the soldering phase. Reverse pulse plating uses precisely controlled reverse current pulses to remove the excess of the material during the plating process [3]. Thus, in many cases there are difficult to obtain satisfactory results with the conventional widespread processes due to the wide range of factors. One of the significant aspects is the correct preparation of hole's surface immediately before copper plating. In fact, various specialized chemical solutions (e.g. solution of copper sulphate, calcium hypophosphite and ammonium hydroxide [4]) can be used for the activation of surfaces, but the still existing problem consists in obtaining homogenously activated/covered surface of plated holes.

In this paper we introduced an innovative method of copper plating, primarily focussed on the preparation of sample surface before the plating process. Newly formulated method was based on the utilization of reverse pulse plating, in which the main innovation is brought by the usage of lower ambient pressure during the activation of surface. In the case of the through-hole plating process, the necessity to prepare/activate the surface of drilled holes before the copper layer could be successfully grown on the walls of the holes with the aid of electrical energy. This process is an extremely important for the creation of conformal layers and its uniformity that subsequently facilitate the adhesion and growth of the copper coating [3].

As it was mentioned before, during the activation of nonconducting surface (drilled holes) the introduction of some additional parts of this process could be helpful. One of such innovations of the process is the addition of lower pressure 
for better protrusion of activator into the holes specified with a smaller diameter. It is well-known fact that low vacuum decreases surface tension of liquids and causes expansion of gasses. It simply forces the air trapped in smaller holes to escape and activator solution penetrates inside [5].

Other concept is using the ultrasound transferred to the test vehicle. An ultrasonic wave is reflected when it strikes an interface between materials with different speeds of sound (acoustic impedance). Furthermore, an interface between materials with a larger difference in acoustic impedance reflects ultrasonic waves more strongly. The smaller difference in acoustic impedance reflects them less strongly and lets part of them travel through [6].

\section{Experimental}

\subsection{Equipment}

Copper plating unit was provided by LPKF MiniContac RS (blue box in the middle of Fig. 1), which consists of four chambers for cleaning (two chambers), activation and copper plating. Those chambers were used for samples fabrication. The unit works as the reverse-current pulse method for cooper plating. This method, reverse pulse copper plating, makes it possible to achieve sufficient parameters for conformal coating of copper during plating.

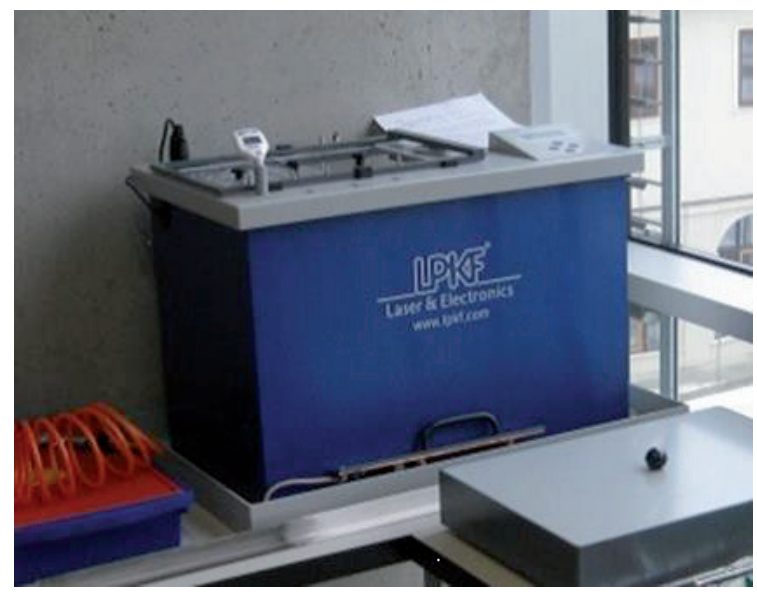

Fig. 1 MiniContac RS

Innovation in this process was the construction of vacuum chamber (Fig. 2) for the activator solution, which allows more reliable activation process of holes with smaller diameters than is common. Chamber has two parts prepared from special, chemical-resistant, plastic material. The first part is the chamber itself and the second part is the cover with the fitting for vacuum hose (Fig. 2). This chamber was also used during the activation procedure involving the ultrasound waves. As reference samples, there was used MiniContac RS built-in activation chamber.

For investigation of plated hole's reliability, resistance test method was used (conforming to IPC-TM-650 standard - test method 2.2.13.1). Figure 3 is showing the fixture used for measurement. Pictures on the right top corner contains details of the measurement pins. Two pins are placed from top side (Fig. 3 top red square) and two from bottom (Fig. 3 - bottom red square). This arrangement is due to the usage of pins for four-point method (two pins for current, two pins for voltage). Measured pattern of test vehicle will be placed between top and bottom pins. Voltage of the tested plated hole will be observed as response on current load $(100 \mathrm{~mA})$ during the analysis procedure. Then resistance was calculated based on the obtained values.

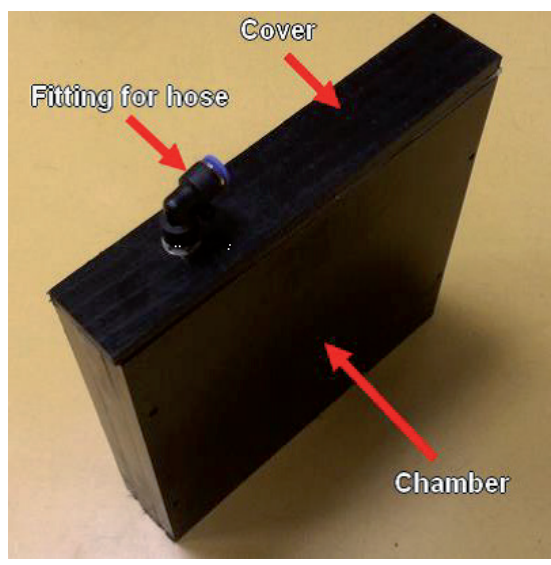

Fig. 2 Vacuum chamber for activation solvent.

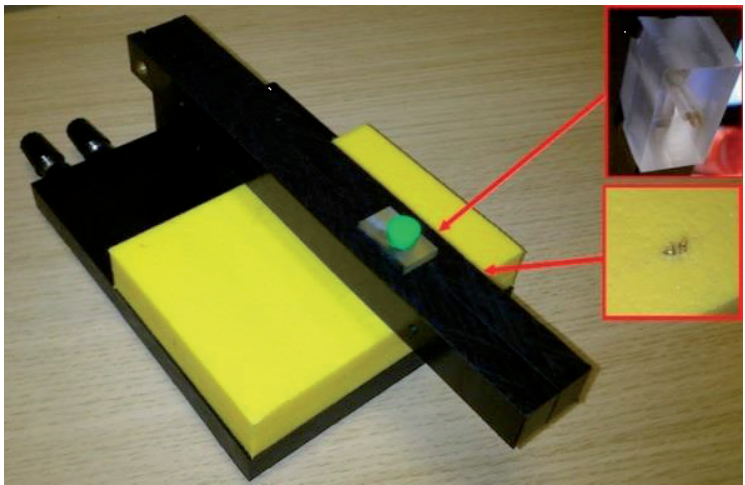

Fig. 3 Measurement fixture

Test vehicle can be seen on the Fig. 4. Dimension of the test coupon is approximately $70 \times 13 \mathrm{~mm}$. Test pads were fabricated on both sides of the $\mathrm{PCB}$ and these are connected to the top-bottom connecting holes (vias). Diameter of holes falls into the range of $0.2-1.5 \mathrm{~mm}$. Holes with different diameters are positioned uniformly for better control of plating process. Two rows of holes with mirrored sequence to each other. Base material used during the experimentation was FR4 substrate that was plated on both sides by $18 \mu \mathrm{m}$ of copper.

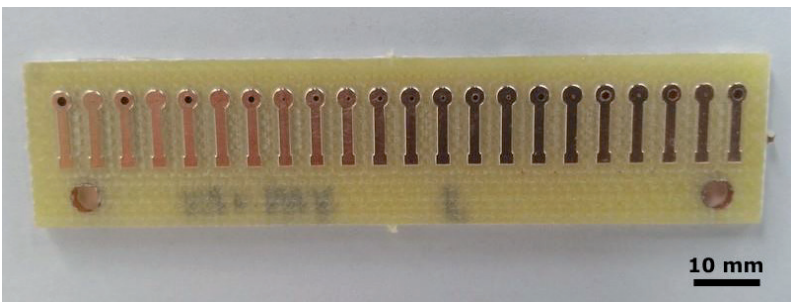

Fig. 4 Test sample 


\subsection{Process of sample fabrication}

Table 1 shows the initial configuration of parameters as these were selected for the individual phases of throughhole plating process. Due to that, samples were prepared for four cases of surface activation.

Table 1 Parameters of plating process.

\begin{tabular}{llll}
\hline Phase & Chemicals & Time (min.) & Other parameter \\
\hline Cleaning 1 & LPKF Cleaner 110 & 10 & Temperature $60^{\circ} \mathrm{C}$ \\
Cleaning 2 & LPKF Cleaner 210 & 10 & \\
Activation & LPKF Activator 310 & 20 & \\
Plating & $\begin{array}{l}\text { LPKF Copper Plater } \\
\text { 400; Shine 400 }\end{array}$ & 40 & Current 2A \\
\hline
\end{tabular}

Samples were fabricated by standard method (Standard sample) and optimized methods (sample fabricated with using vacuum, ultrasound and vacuum with ultrasound). Activation process was performed in the following variants:

- Standard process (reference sample) - shaking with sample in activation bath.

- Ultrasound - sample was attached in chamber with activation solvent and the chamber was placed into water (for better transfer of ultrasound waves) with ultrasound source. Frequency of ultrasound was around $25 \mathrm{kHz}$.

- Vacuum - sample was attached in vacuum chamber with activation solvent (Fig. 2). Vacuum had $0.8 \mathrm{~atm}$.

- Vacuum with ultrasound - sample was attached in vacuum chamber with activation solvent and the chamber was placed into water (for better transfer of ultrasound waves) with ultrasound source.

Four samples were prepared for each test case scenario. One sample contains up to 8 holes of the same diameter. The thickness of the deposited copper has reached approximately $3 \mu \mathrm{m}$ for the first package of samples. This particular thickness was used for the detection of the very first defects. Second package of samples was made with $20 \mu \mathrm{m}$ of plated copper. This thickness was selected for verification obtained results from samples with $3 \mu \mathrm{m}$ plated copper and also will be used for next work on reliability investigation. Values of copper thickness were measured from cross-sections.

\subsection{Test methods}

According to the specification of IPC-TM-650 test method 2.2.13.1, theoretical value of resistance was calculated for corresponding thickness of plated copper ( $3 \mu \mathrm{m}$ and $20 \mu \mathrm{m})$ using the following equation [7]:

$$
R=\frac{0.21548 \times 10^{-6}(T)}{D t-t^{2}}
$$

where $\mathrm{T}$ is the thickness of $\mathrm{PCB}, \mathrm{D}$ is the diameter of hole and $t$ is the thickness of plated copper.

According to that standard, the resistance measurement method was used. It helped to identify the number of failures and the conductivity of vias. Failure criteria was established as the resistance higher than $\pm 10 \%$ deviation from modus of measured values.

X-Ray and cross-sections methods were used for in-depth inspection. These methods helped to get a better understanding of defects that occurred during the process of activation.

\section{Results}

Measurement of resistance was used for establishing the failure rate as the first. Insufficient plating was identified only for hole diameters $0.2 \mathrm{~mm}$ and $0.4 \mathrm{~mm}$. Due to that observation, the further investigation throughout this work was focused only on these two hole diameters.

Chart of number of failures for $0.2 \mathrm{~mm}$ and $0.4 \mathrm{~mm}$ holes with $3 \mu \mathrm{m}$ copper thickness is shown in Fig. 5. Graph shows the influence of vacuum and vacuum with ultrasound. The number of unplated holes was lower than for reference samples. Only samples activated with ultrasound showed more failures than the other ones.

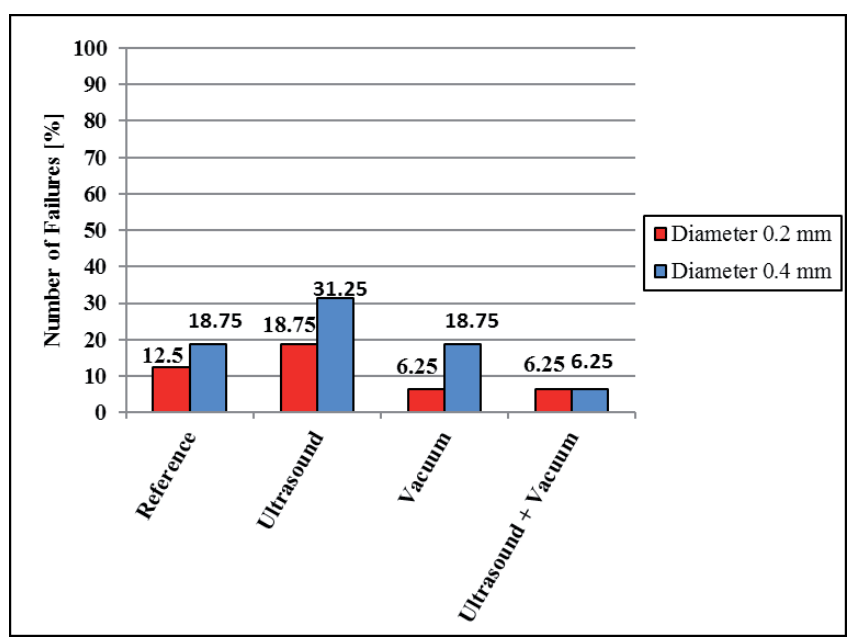

Fig. 5 Number of failures - holes of diameter $0.2 \mathrm{~mm}$ and $0.4 \mathrm{~mm}$ with $3 \mu \mathrm{m}$ thickness copper.

The next graph (Fig. 6) shows the number of failures for both sizes of holes with $20 \mu \mathrm{m}$ copper thickness plated on hole's edges. Trends of number of failures are the same as in Fig. 5. However, $0.4 \mathrm{~mm}$ holes had less failures than $0.2 \mathrm{~mm}$ holes.

Figure 7 shows the average values of measured resistance for each activation process and for the diameter of holes $0.2 \mathrm{~mm}$ and $0.4 \mathrm{~mm}$. Theoretical/calculated (equation (1)) values of resistance were $2.5 \mathrm{~m} \Omega$ for $0.2 \mathrm{~mm}$ holes and $1.2 \mathrm{~m} \Omega$ for $0.4 \mathrm{~mm}$ holes. These two values are not entirely corresponding with measured ones. The equation is calculated for $100 \%$ conductivity of plated copper and its bigger thickness. Therefore, 
measured values are of the same order (ones of $m \Omega$ ). Due to values of standard deviations, there were no effects of the activation process on the resistance of plated through-hole.

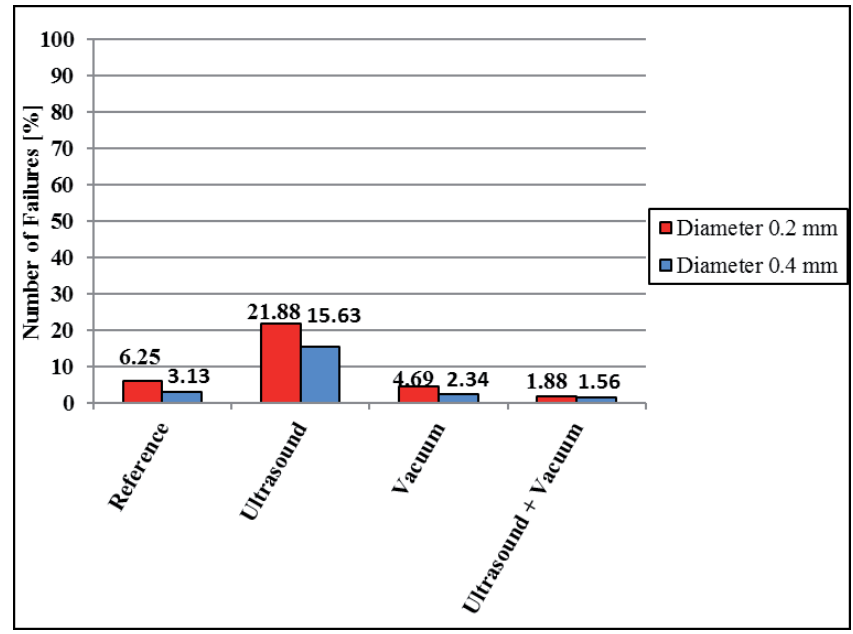

Fig. 6 Number of failures - holes of diameter $0.2 \mathrm{~mm}$ and $0.4 \mathrm{~mm}$ with $20 \mu \mathrm{m}$ thickness copper.

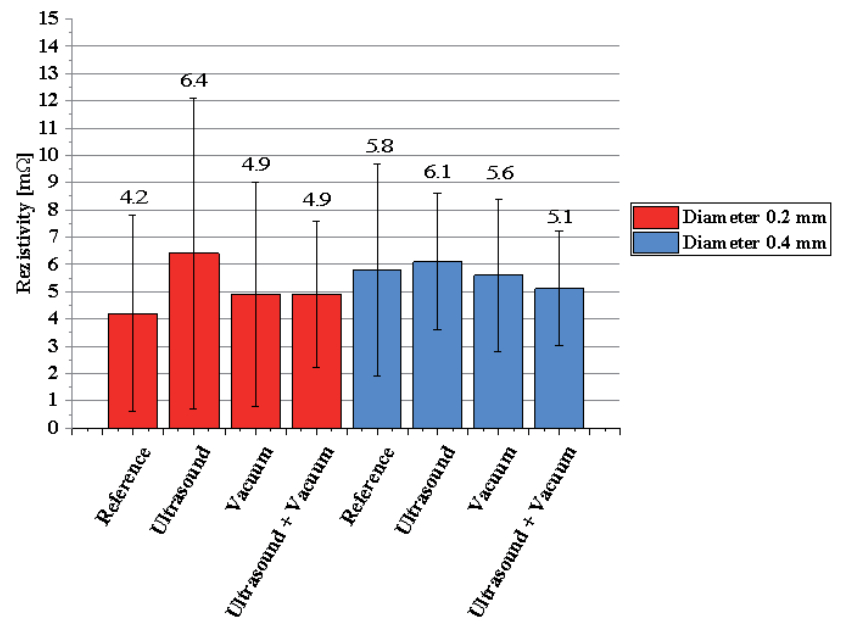

Fig. 7 Measured resistance for $3 \mu \mathrm{m}$ of plated copper (hole diameter $0.2 \mathrm{~mm}$ and $0.4 \mathrm{~mm}$ )

Results for measured resistance of 20 um plated copper is shown in Fig. 8. This graph shows same parameters as graph in Fig. 7, resistance for two diameters of through holes activated with different conditions. For comparison of measurement with theoretical values, there was calculated resistance $0.207 \mathrm{~m} \Omega$ for $0.2 \mathrm{~mm}$ holes and $0.103 \mathrm{~m} \Omega$ for $0.4 \mathrm{~mm}$ holes. Calculated values are not the same due to the same influences as were mentioned in previous paragraph. Also, there was not major influence on the resistivity in dependence on activation method due to values of standard deviations.

Second analysis was provided by X-Ray inspection equipment. Results are presented on the Fig. 9, Fig. 10 and Fig. 11. On the first figure (Fig. 9) is shown unplated through hole of standard sample. This defect was discovered only after the standard activation process. New methods of activation have not had this type of defect, expect method using only ultrasound. Other defect was still occurring after all type of activation processes. Insufficient plated hole is shown on Fig. 10. Figure 11 shows right plated hole of sample for comparison with defective one.

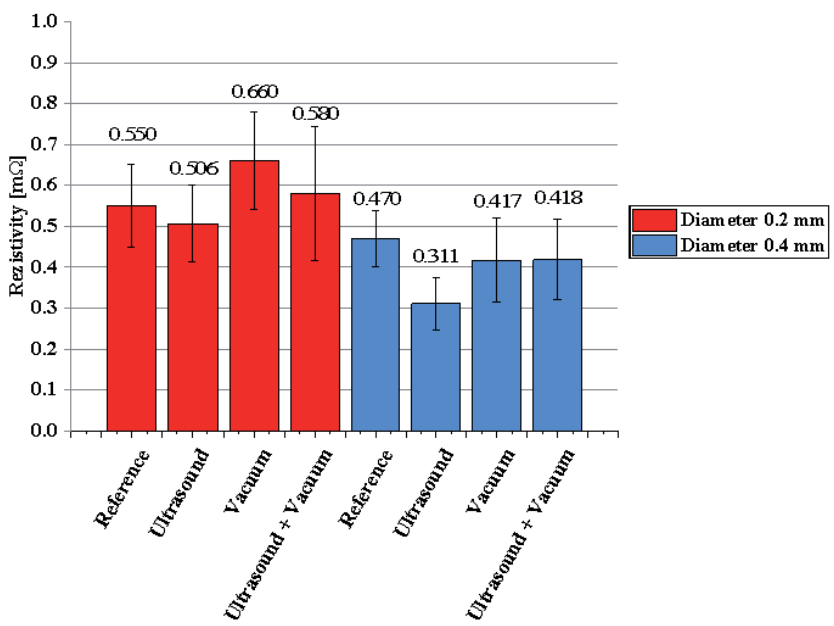

Fig. 8 Measured resistance for $20 \mu \mathrm{m}$ of plated copper (hole diameter $0.2 \mathrm{~mm}$ and $0.4 \mathrm{~mm}$ )

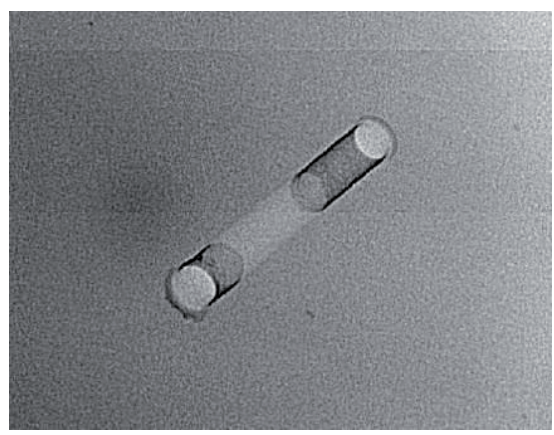

Fig. 9 X-Ray - unplated hole (diameter $0.2 \mathrm{~mm}$ ).

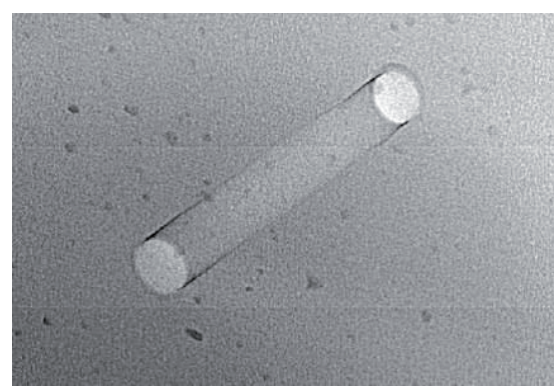

Fig. 10 X-Ray - insufficient plated hole (diameter $0.2 \mathrm{~mm}$ ).

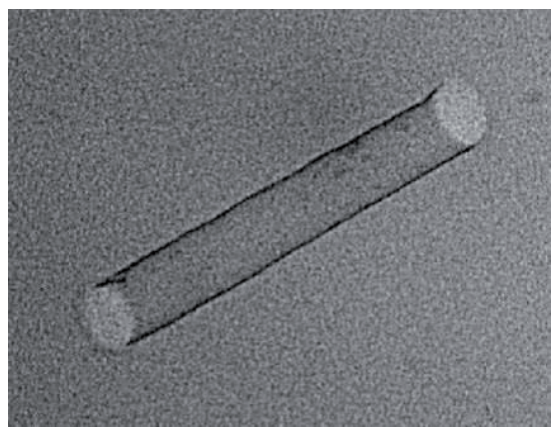

Fig. $11 \mathrm{X}$-Ray - right plated hole (diameter $0.2 \mathrm{~mm}$ ). 
Cross-sections showed much more details than X-Ray pictures. Fig. 12 shows defect, which was mainly connected with the standard activation process used before copper plating, also shown on the Fig. 9. New methods helped to eliminate this type of defect. Figure 13 (right plated hole) was used for comparison of the fully plated hole with defective one.

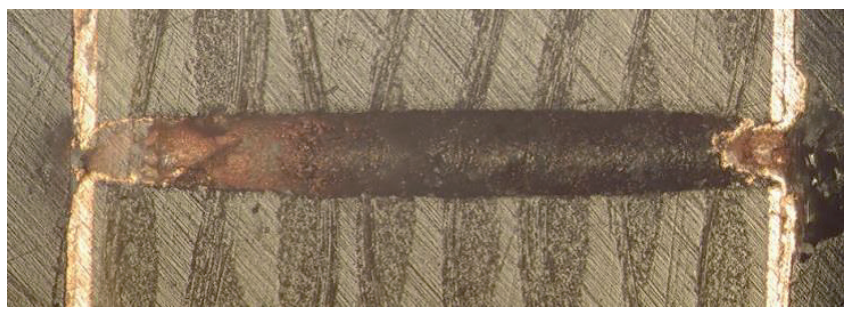

Fig. 12 Cross-section of unplated hole (diameter $0.2 \mathrm{~mm}$ ).

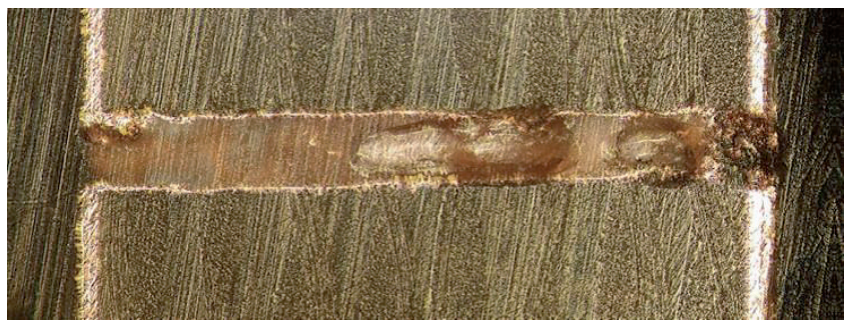

Fig. 13 Cross-section of right plated hole (diameter $0.2 \mathrm{~mm}$ ).

Next figure (Fig. 14) shows detail on plated copper layer. There are marked carbon layer, which was deposited during activation process, and plated hole. Thickness of carbon layer depends on the process of drying after sample was removed from activation bath. In the base, that layer did not affect any parameters observed in this work. However, this information could be important in future investigation. Plated copper layer is also shown in hole's edge. There is shown grown copper layer. This indicates the correct process of plating.

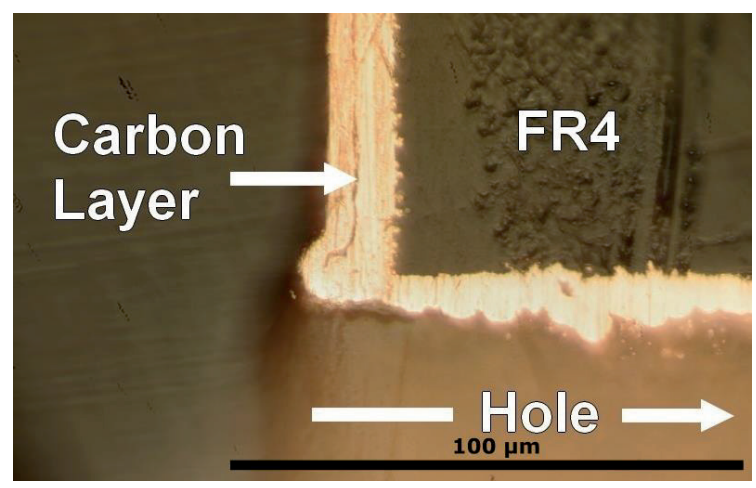

Fig. 14 Detail on plated copper in through-hole's edge

\section{Discussion}

Difference between 0.2 and $0.4 \mathrm{~mm}$ diameter holes $(3 \mu \mathrm{m}$ of plated copper), where $0.4 \mathrm{~mm}$ holes had bigger amount of failures than $0.2 \mathrm{~mm}$, could be explained by thinner thickness of plated copper. Some holes could be sufficiently plated. Results for samples with $20 \mu \mathrm{m}$ of copper show that supposed behavior.
The obtained results showed a positive effect of vacuum and vacuum with ultrasound. Reducing the number of defects in the two methods is due to the lower pressure, ie. reduced surface tension of the activating solution, and also increased the expansion of gases. Due to this behavior, there is a better penetration of the solution into the holes with a smaller diameter. Surprisingly, the best results in the activation of the vacuum in the presence of ultrasound. The same behavior was observed in moulding process of housing process in PCB manufacturing process [9].

Conversely, when using only ultrasound increased number of defects with insufficient metal coating. Based on X-ray images of plated holes, there can be concluded that no improvement in throughput of the activation solution into holes with a diameter of $0.2 \mathrm{~mm}$. The similar effect was described in paper which deals with process of electroless copper plating on carbon nano foams [10]. Ultrasound was acting on liquid solvent. Due to that, liquid could not get to small cavities. Article [11] describes enhanced method of copper plating of blind-vias with using ultrasonic agitation, but in this case, the basics of plating process are different from process of activation.

Quality of plated copper was good in according to reference [12]. Formed copper crystals were ordered to uniform grain structure.

Next work in this topic will be focused on reliability. Reliability test will be provided thermal cycling in according to standard IPC-TM-650 Method 2.6.26. After thermal cycling, there will be also studied structure with using cross-section method and SEM microscopy.

\section{Conclusion}

Various copper plating methods are known in the printed circuit fabrication industry. Settings of process parameters are depended on the purity of solvents and knowledge and responsibility of operator. Therefore, the using of vacuum or ultrasound during activation process can help to establish new enhanced process of through-hole plating, which is suitable also for prototyping domain. Reliability of copper plated holes activated with the utilization of just vacuum and combination of vacuum and ultrasound were better in comparison of standard method of activation. The best results were achieved with combination of vacuum and ultrasound.

All presented defects was appeared due to activation process, as was mentioned in the beginning of this paper. This hypothesis was approved by this work (X-Ray pictures, cross-sections).

In this paper are described first results of innovated process of copper plating. Performed analysis found a significant influence of vacuum and ultrasound on a number of defects caused during the activation process. 


\section{Acknowledgement}

Funding for this research work was supported through of the Czech Ministry of Education for Brno University of Technology FEKT-S-14-2168 and grant project FEKT/FIT-J-15-2832. Thanks to Sanmina - SCI Czech Republic for opportunity use their X-Ray inspection system.

\section{References}

[1] Shen, F.-Y., Dow, W.-P., Liu, A.-H., Lin, J.-Y., Chang, P.-H., Huang, S.M. "Periodic Pulse Reverse Cu Plating for Through-Hole Filling." ECS Electrochemistry Letters. 2(5), pp. D23-D25. DOI: 10.1149/2.003305eel

[2] Baker Technology Associates Inc. "Why Pulse Plating?" 2015. [Online]. Available from: http://www.bakertech.com/pulse.htm [Accessed: 6th September 2016]

[3] LPKF Laser\&Electronics. 2015. [Online]. Available from: http:// www.lpkfusa.com/RapidPCB/ThroughHolePlating/minicontac_rs.htm [Accessed: 8th April 2016]

[4] Instructables. "Industrial-level quality PCB through hole plating." 2015. [Online]. Available from: http://www.instructables.com/id/Inexpensivemethod-of-industrial-level-quality-PCB/ [Accessed: 6th September 2016]

[5] O'Hanlon, J. F. "A user's guide to vacuum technology." Wiley-Interscience. 1980
[6] NIHON DEMPA KOGYO CO., LTD. "Ultrasound and its properties." 2015. [Online]. Available from: http://www.ndk.com/en/sensor/ultrasonic/basic01.html [Accessed: 6th September 2016]

[7] IPC-TM-650 Test Methods Manual, 2.2.13.1, The Institute for Interconnecting and Packaging Electronic Circuits

[8] IPC-6012B Adendment 1 Qualification and Performance Specification for Rigid Printed Boards, The Institute for Interconnecting and Packaging Electronic Circuits

[9] Gielen, P., Sillen, R., Puik, E. "Low cost environmentally friendly ultrasonic embossed electronic circuit board." In: Electronic System-Integration Technology Conference (ESTC), 2012 4th, Amsterdam, Netherlands, Sept. 17-20, 2012, pp. 1-7. DOI: 10.1109/ESTC.2012.6542058

[10] Zhai, L., Liu, X., Li, T., Feng, Z., Fan, Z. "Vacuum and ultrasonic coassisted electroless copper plating on carbon foams." Vacuum. 114, 2015. pp 21-25. DOI: 10.1016/j.vacuum.2014.12.005

[11] Chen, Q., Wang, Z., Cai, J., Liu, L. "The influence of ultrasonic agitation on copper electroplating of blind-vias for SOI three-dimensional integration." Microelectronic Engineering. 87(3), pp. 527-531. 2010. DOI: 10.1016/j.mee.2009.06.035

[12] Konstantin, G., Kück, H., Münch, R. "A novel manufacturing technology for tensile test specimens for the characterization of copper in plated through holes (PTH)." In: Electronics Packaging Technology Conference (EPTC 2013), 2013 IEEE 15th, Singapore, Dec. 11-13, 2013, pp. 33-36. DOI: $10.1109 /$ EPTC.2013.6745678 